\title{
Promoviendo la autorregulación en la resolución de problemas de física
}

\section{Promoting self-regulation in solving problems in physics}

\author{
Julià Hinojosa ${ }^{1}$ • Neus Sanmartí ${ }^{2}$
}

Resumen: La autorregulación es una estrategia eficaz para desarrollar la competencia en aprender a aprender, al mismo tiempo que la científica. En este trabajo se presenta una actividad orientada a promover el desarrollo de la capacidad de los alumnos de 16 y 17 años de autorregular los procesos de resolución de problemas de física, y de la autoevaluación del mismo proceso. Los análisis de los datos de su aplicación muestran, por una parte, que mejoran los resultados de los estudiantes, así como la valoración positiva que hacen del proceso, y, por otra parte, su influencia en el cambio en el quehacer diario del profesor.

Palabras clave: Autorregulación. Resolución de problemas. Enseñanza de la física. Base de orientación. Autoevaluación.

\begin{abstract}
Self-regulation is an effective strategy to develop competence in learning to learn, and in scientific literacy. This paper presents an activity aimed to promote the development of the ability of students 16 to 17 years old to self-regulate the processes of solving physics problems, and self-assessment activity as the process itself occurs. The analysis of the data from its application shows, firstly, that students improve their results and the positive assessment made of the process and, furthermore, its influence on the change in the daily work of the teacher.
\end{abstract}

Keywords: Self-regulation. Problem solving. Physics teaching. Scaffolding. Self-assessment.

\footnotetext{
${ }^{1}$ Escola Pia Sarrià-Calassanç, Ciencias Experimentales, Barcelona, Cataluña, España. E-mail: <julia.hinojosa@ escolapia.cat>

${ }^{2}$ Universidad Autonoma de Barcelona, Barcelona, Cataluña, España.
} 


\section{Introducción y objetivos}

La resolución de problemas desempeña un papel crucial en el currículo de las ciencias (LORENZO, 2005; McDERMOTTT, 1998). Puig y Cerdán (1988) resaltan que la tarea de resolver problemas es una tarea privilegiada para el aprendizaje, ya que normalmente es la actividad de producción de conocimiento donde se aplican los adquiridos a nuevas situaciones, es decir, donde se promueve la transferencia del aprendizaje. Se concibe normalmente como una actividad generadora de un mecanismo a través del cual, quien aprende, combina información teórica (conceptos, leyes, principios), procedimientos (por ejemplo, calculo aritmético y algebraico, control de variables, emisión de hipótesis, interpretación de gráficos, etc.) y, finalmente una actitud favorable hacia la tarea y/o hacia la disciplina en cuestión. Es decir, que conlleva la convergencia de las tres dimensiones básicas del conocimiento y su activación (PERALES, 2000). La literatura sugiere que el éxito en la resolución de problemas depende de la combinación de un potente conocimiento de la materia, conocimiento de las estrategias de resolución de problemas y de componentes actitudinales (JONASSEN, 2000).

En general, la mayoría de las propuestas para la enseñanza de la resolución de problemas se articulan, expresadas de una u otra forma, alrededor de las cuatro fases utilizadas por Polya (1987): comprensión del problema, concepción de un plan, ejecución del mismo y visión retrospectiva o revisión de los resultados. Reif (1995) las concretó en: descripción y análisis del problema, construcción de una solución, y control de la solución y otros autores las explicitaron de forma parecida (HEYWORTH, 1998; PERALES, 2000; SCHRAW; CRIPPEN; HARLEY, 2006). El análisis de esas etapas mostró que los componentes de la resolución de problemas son muy complejos para ser aprendidos a partir de ejemplos y de la práctica. La capacidad de resolver problemas depende no solamente del aprendizaje de los procedimientos sino también de la capacidad de hacer un uso apropiado de los conocimientos de física asociados a cada tipología de problemas (REIF, 1985).

Las investigaciones en didáctica de las ciencias ponen de manifiesto las deficiencias de los modelos de instrucción habituales (GERACE; BEATTY, 2005). La educación tradicional forma estudiantes que en muchas ocasiones se encuentran poco motivados, aburridos de la forma en la que aprenden. Memorizan gran cantidad de información que olvidan en poco tiempo y se vuelve irrelevante en el mundo exterior al centro educativo (GIL et al., 1999). Por consiguiente, no es de extrañar que mejorar las habilidades de los estudiantes en la resolución de problemas continúe siendo un objetivo principal de los profesores e investigadores en la didáctica de las ciencias (SOLAZ-PORTOLÉS; SANJOSÉ-LÓPEZ, 2008). Muchas son las líneas de investigación orientadas a superar las dificultades de los que aprenden. Por ejemplo, las orientadas a plantear problemas de acuerdo con las metodologías científicas (GIL; MARTÍNEZ-TORREGROSA; SENENT, 1988); o las que analizan las diferencias entre expertos y novatos (BUTELER; GANGOSO, 2008); o las que se fijan en la contextualización de los problemas para que respondan a situaciones reales, problematizadas - ABP y otras (PERALES, 2000).

Nuestro estudio arranca de la búsqueda de propuestas que ayuden a los alumnos de 16-17 años a afrontar la resolución de problemas cotidianos en general y, en particular, de física a partir de promover el desarrollo de estrategias metacognitivas. Normalmente, los estudiantes ya han construido sus propias estrategias para conseguir resolver un problema de cualquier 
índole, pero frecuentemente estas estrategias no son idóneas tanto debido a dificultades de índole metodológica y matemática, como de mala activación de los conocimientos de física, impidiendo ser eficaz y al mismo tiempo eficiente.

El trabajo llevado a cabo con los estudiantes que se presenta en este escrito ha buscado promover que sean capaces de autorregularse, a partir de reflexionar metacognitivamente sobre el proceso que aplican para resolver el problema y sobre cómo utilizan los saberes aprendidos. Este estudio se centra en la descripción y fundamentación de la estrategia aplicada y en el análisis de los resultados obtenidos relacionados con la comprobación de las mejoras en el aprendizaje.

\section{La autorregulación como componente básico de todo aprendizaje competencial}

Schraw, Crippen y Harley (2006) señalan que el aprendizaje autorregulado tiene tres componentes principales: la cognición, la metacognición y la motivación. La cognición incluye las habilidades necesarias para codificar, relacionar, jerarquizar, memorizar y recuperar información; la metacognición integra estrategias que permiten a los estudiantes comprender y controlar sus procesos cognitivos; y la motivación incluye las creencias y actitudes que afectan el uso y desarrollo de las habilidades cognitivas y metacognitivas. Por tanto, una actividad de resolución de problemas debería orientar a los estudiantes en la aplicación de habilidades básicas y necesarias para dicha resolución y en promover su autoevaluación desde un punto de vista metacognitivo. Es de suponer que este tipo de actividades posibilitarán aprender más y mejor y, en consecuencia, estimularán la disposición del alumnado hacia el aprendizaje.

Cometer errores a lo largo de un proceso de aprendizaje es algo totalmente normal e inherente al mismo proceso de aprender (BUNGE, 2013). Sin embargo, los profesores constatamos que hay unos estudiantes que son más capaces que otros de reconocer los obstáculos que han de superar al resolver un problema y de tomar decisiones para afrontarlos. La diferencia básica es que unos saben evaluarse (regularse), mientras que otros han desarrollado sistemas para aprender muy poco eficientes.

Cada alumno, cada persona, tiene un sistema personal de aprender que ha ido construyendo progresivamente de manera autónoma, básicamente a partir de los estímulos que recibe en la escuela y en su entorno familiar y social. Por ejemplo, unos chicos y chicas tienden a refugiarse en la repetición y en la mecanización, y piden ayuda externa constantemente, mientras que otros buscan entender por qué no entienden o por qué no les sale bien. Por supuesto, los resultados de unos y otros a largo plazo son muy diferentes.

Aprender de forma significativa es necesariamente un proceso de autorregulación de tipo metacognitivo (SCHOENFELD, 1987). Pero ¿cómo desarrollar un sistema de aprendizaje que permita aprender a aprender, es decir, a autorregularse? A partir de la Teoría de la Actividad promovida por Leontiev se considera que es necesario (SANMARTÍ, 2007; SANMARTÍ; JORBA, 1995):

- Representarse la categoría del problema y los motivos y los objetivos de su resolución, es decir, poder responder a cuestiones como: ¿qué tipo de actividad-problema debo resolver? ¿por qué realizar esta actividad? ¿Qué aprenderé con ello? 
En los trabajos de Chi, Feltovich y Glaser (1981) y Chi (2006), donde se identifican las diferencias en las formas como los expertos y los novatos resuelven los problemas de física, queda expuesta una tendencia de los expertos a categorizar los problemas según una "estructura profunda", mientras que los principiantes tienen la tendencia a categorizarlos a partir de características superficiales. Los expertos no sólo tienen más conocimientos, sino que los han organizado para ser útiles y saben activarlos de forma idónea.

Muchos autores sostienen que un sujeto comprende un texto sólo si es capaz de construir un modelo situacional adecuado del mismo, o sea, si es capaz de relacionar la información textual con el conocimiento previo del lector (GÓMEZ-FERRAGUD, 2014; SÁNCHEZ, 2011). Ese modelo inicial le permite al sujeto comenzar a resolver el problema. Una de las dificultades más importantes que los alumnos constatan es que al leer el enunciado no activan a qué categoría de problemas pertenece el que se les propone resolver, es decir, con qué tipología o clase conecta de los que han trabajado anteriormente. La no activación se debe a que no simplifican bien la realidad para reflejarla al construir el modelo del problema (COOK; REICHARDT, 1986; GUISASOLA et al., 2011; SANJOSÉ et al., 2007). Esta dificultad en construir la representación de carácter concreto, asociado al mundo real, puede conducir a obstáculos en la construcción de la representación abstracta del problema, necesaria para resolverlo algebraicamente (GÓMEZ-FERRAGUD; SOLAZ; SANJOSÉ, 2013). Por otra parte, generalmente, el objetivo de los estudiantes es aprobar más que reconocer el campo de aplicación-utilidad del problema y sin reconocer qué están aprendiendo y su finalidad, es muy difícil que puedan autorregularse.

- Anticipar y planificar las operaciones necesarias para llevar a cabo la acción, es decir, poder responder a cuestiones como: ¿qué estrategia o estrategias se pueden adoptar para resolver la situación planteada?, ¿cuál de estas estrategias es la más adecuada para realizar la tarea propuesta?, ¿qué operaciones es necesario realizar para conseguirlo y por qué?, ¿en qué orden?, ¿qué conocimientos son necesarios?, ¿cuál es el resultado esperado?

Cómo indican todos los estudios (DUFRESNE et al., 2000; SCHOENFELD, 1987), las personas expertas en resolver problemas dedican mucho tiempo a planificar y poco a ejecutar, mientras que las novatas hacen todo lo contrario. En esta línea, las bases de orientación (NUNZIATI, 1990) son unos instrumentos idóneos para estimular que los estudiantes se habitúen a anticipar cómo resolver un problema (o realizar distintos tipos de tareas), y a regular su aplicación y, en general, cualquier tipo de organizador gráfico. Pero también es cierto que habitualmente los profesores calificamos (damos valor) a los resultados y no a la planificación, por lo que se estimula a los que aprenden a buscar dichos resultados sin desarrollar habilidades orientadoras.

- Finalmente será necesario identificar los criterios de evaluación que permiten decidir si al aplicar la planificación diseñada se está haciendo de forma adecuada. Esta regulación puede pasar por revisar la categoría con la que se ha conectado el problema a resolver o sus objetivos, o por replantear los distintos ítems de la planificación diseñada (base de orientación), aspectos en los que habitualmente no suelen pensar los estudiantes. Generalmente les es más fácil analizar el resultado final y, en caso que éste no sea lógico, revisar la ejecución del problema comprobando si se ha realizado tal como se había previsto, pero pocas veces son capaces de pensar en replantear dicha planificación. 
Las habilidades que ayudan a comprender, controlar y evaluar los procesos cognitivos cobran especial interés en el ámbito de la resolución de problemas. Comprender el problema para planificar estrategias, controlarlas y evaluar los resultados son las acciones que permiten al resolutor desenvolverse con facilidad en el espacio de resolución. Por tanto es un reto para el profesorado conseguir que los estudiantes adquieran una buena capacidad de autorregularse de manera efectiva, lo que comporta que lleguen a apropiarse de los objetivos y de los criterios de evaluación del profesorado y a tener un buen dominio de las capacidades de anticipación y planificación de la acción e implica incorporar estos aspectos como objetivos prioritarios de aprendizaje. Los estudios realizados (SANMARTÍ; SIMÓN; MÁRQUEZ, 2006) demuestran que no es tarea fácil, ya que los profesores también tenemos nuestras rutinas. Truyol y Gangoso (2010) estudian el modo en que los enunciados de los problemas promueven el desarrollo de competencias propias de los físicos profesionales, como la construcción de un modelo físico a partir de un problema basado en la realidad. También, a partir de la actividad diseñada en esta experiencia, se intenta encontrar más estrategias para responder a este reto.

\section{Actividad aplicada y su justificación}

Para ayudar a los estudiantes a mejorar sus estrategias y a aplicarlas de forma coherente, a nivel del departamento de ciencias experimentales del centro experimentador se diseñó una base de orientación para promover en el alumnado una mejora en su proceso de resolución de problemas (Cuadro 1). El significado de esta base de orientación, que explicita y es el extracto natural de muchas de las prácticas habituales de enseñar a resolver problemas, se consensua con los estudiantes y, a partir de ella, se les estimula a revisar las estrategias que tienden a aplicar de forma más o menos rutinaria y a dar sentido a cada una de las acciones. En esencia, estamos redefiniendo nuestro papel como instructores y pasamos a ser más bien guías u orientadores del aprendizaje de los estudiantes.

La reflexión con los estudiantes sobre el uso de esta base de orientación se fundamenta en la importancia de dedicar tiempo a la anticipación y planificación de la acción, y en cómo la base de orientación consensuada puede ser útil para pensar antes de hacer, con la finalidad de ser más eficaces resolviendo problemas (BUTELER; GANGOSO, 2008; GERACE; BEATTY, 2005; LORENZO, 2005). Posteriormente se anima a los estudiantes a ponerla en práctica. Primero la utilizan como guía para resolver problemas que forman parte de sus deberes, y después, cuando ya empiezan a interiorizarla y a rutinizar su uso, pasa a ser una estructura a aplicar también en la resolución de problemas en los exámenes.

Para estimular su aplicación de forma eficaz, se establece un fórum en la plataforma educativa virtual de clase. Se sube a la plataforma, escaneado, el documento que reproduce el proceso aplicado por un alumno cualquiera en la resolución de un problema y, seguidamente, se abre un fórum donde los compañeros escriben tanto sus críticas y propuestas de mejora, como sus autocríticas, siempre con referencia a los ítems de la base de orientación consensuada que sirve de apoyo. Rápidamente se establece una conversación argumentada sobre las diferentes posibilidades. Este fórum, para cada problema tipo, se realiza aproximadamente durante una semana. 
Cuadro 1. Base de orientación para la resolución de problemas de ciencias

\begin{tabular}{|l}
$\begin{array}{l}\text { Nombre: } \\
\text { Nivel: }\end{array}$ \\
$\begin{array}{l}\text { Asignatura: } \\
\text { Curso: }\end{array}$ \\
$\begin{array}{l}\text { Problema (Enunciado del problema) } \\
\text { 1. Leer de forma comprensiva (lectura en silencio y atención). } \\
\text { 2. Representar el problema situando los datos, ya sea mediante un esquema, un dibujo o } \\
\text { simplemente un listado. Este apartado ha de servir para autoevaluar y regular la comprensión } \\
\text { lectora. } \\
\text { 2.1. Adecuar las unidades al sistema internacional. } \\
\text { 2.2. Identificar las variables del proceso: conocidas y desconocidas. } \\
\text { 3. Identificar el proceso que tiene lugar. Pensar con qué tipología de problemas de física se } \\
\text { relaciona. } \\
\text { 4. Planificar la resolución seleccionando las leyes que gobiernan el proceso. En la mayoría de los } \\
\text { casos plantear ecuaciones mediante de las leyes, es decir, pasar la información lógica del texto a un } \\
\text { lenguaje algebraico a través del modelo. Una ecuación por incógnita. } \\
\text { 5. Formular una anticipación de la solución, aunque sea cualitativa de lo que pasará. } \\
\text { 6. Solucionar matemáticamente las ecuaciones. } \\
\text { 7. Analizar lógicamente la solución para detectar posibles errores en la aplicación del conocimiento } \\
\text { de física en la planificación o incoherencias. } \\
\text { 8. Expresar la solución final con claridad indicando las unidades correspondientes en SI. Escribir } \\
\text { una frase que incluya la solución. }\end{array}$ \\
\end{tabular}

Fuente: elaborado por los autores.

Del análisis de la discusión en fórum se pueden identificar los argumentos de los alumnos y valorar el grado de asimilación de los contenidos conceptuales, procedimentales e, incluso, actitudinales, así como sus principales dificultades y obstáculos a superar. El mismo análisis también ayuda a reconocer deficiencias en la explicación y proceso de enseñanza aplicado por el docente y revisar, así, su práctica. Más información sobre el análisis del funcionamiento de este tipo de fórum se puede encontrar en Hinojosa y Sanmartí (2011).

Para promover que los estudiantes identifiquen las razones de sus dificultades cuando se enfrentan a la resolución de un problema y también para que el profesorado podamos comprender la lógica y las estrategias que ellos aplican, se diseñó un guion de autoevaluación (Tabla 1), fundamentado en la base de orientación de resolución de problemas (Cuadro 1).

Cada alumno autónomamente lo completa para cada problema (Pi) poniendo un 1 en las celdas correspondientes a los ítems en los que ha encontrado dificultades después de realizar distintas pruebas o exámenes y más tarde, voluntariamente, se analiza con ayuda del profesor. Se les pide que reflexionen sobre la causa principal y no marquen más de dos causas para focalizar el problema. 
Tabla 1. Ficha de autoevaluación de problemas de ciencias. Entre paréntesis se han relacionado las categorías de error con los puntos de la base de orientación de resolución de problemas del Cuadro 1

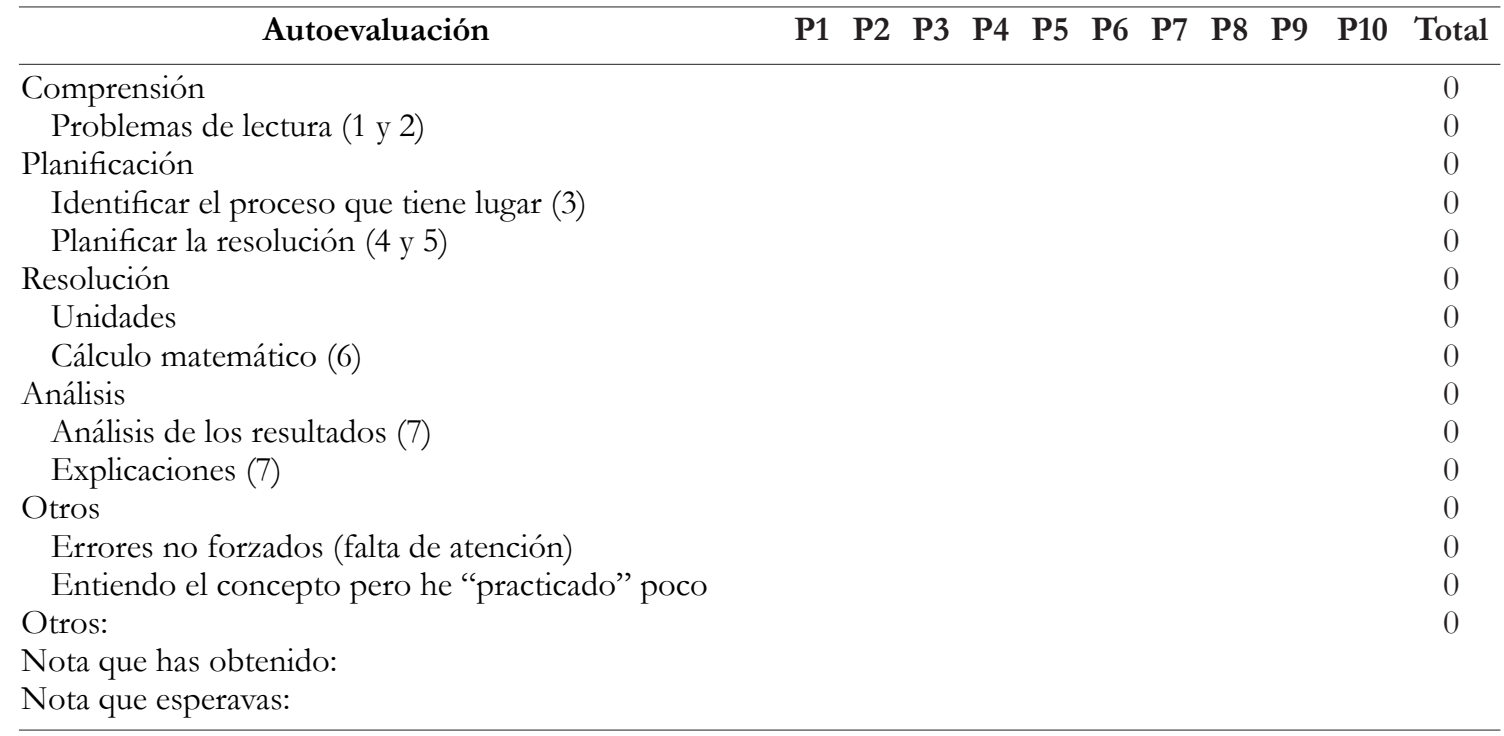

\section{Autoevaluación}

Es posible que... (cada alumno escribe sus propios comentarios a partir de los sugeridos)

\section{Comprensión \\ Problemas de lectura (1 y 2 )}

\section{Planificación} Identificar el proceso que tiene lugar (3)

Planificar la resolución (4 y 5)

Resolución

Unidades

Cálculo matemático (6)

Análisis

Análisis de los

resultados (7)

Explicaciones (7)

Otros

Errores no forzados (falta de atención)

Entiendo el concepto pero he "practicado" poco

Otros:

Nota que has obtenido:

Nota que esperabas:
Se tiene que estar muy concentrado en la lectura y en tanto que sea posible has de revivir el problema mentalmente de manera que cuando dibujes lo que explica el problema te sirva como una autoevaluación de la comprensión de la lectura.

Al leerlo no "activas" a que categoría de problemas pertenece el que tienes delante, con que tipología conecta de los que ya has trabajado. Es aquello que dicen los expertos, este problema "va de...". Lees, pero no "conectas".

Los buenos "resolvedores" de problemas se pasan más tiempo planificando que escribiendo. Has de entender la importancia de pensar antes de hacer.

Atención, todas tendrían que estar en S. 1.

Matemáticas. Quizá te falta práctica?

Tendrias que preguntarte siempre si el resultado tiene sentido.

Tendrías que justificar a través de un modelo cualquier resultado que presentes.

Atención. Intenta estar concentrado y no te distraigas.

La agilidad, en el tiempo, vine dada por la falta de práctica.

Fuente: elaborado por los autores. 
En el guion, como se puede observar, los alumnos se autoevalúan problema a problema siguiendo básicamente como referencia la estructura de la resolución de problemas ya indicada. El guion se crea en una hoja de cálculo y en la columna (Total) se refleja la suma total de veces que han tenido dificultades en cada uno de los ítems expresados. En relación a cada una de las dificultades se indican posibles causas a tener en cuenta y consejos para promover la reflexión, aunque los estudiantes también pueden aportar sus propias ideas.

Uno de los resultados cualitativos de más interés fue que los alumnos constataban que al leer el enunciado no activaban a qué categoría de problemas pertenecía el que tenían que resolver (idea que forma parte del punto 3 de la base de orientación, Cuadro 1). Por ello, en la última versión de la base de orientación se introdujo una pregunta para promover esta conexión y la autorregulación del propio alumno: "Pensar con qué tipología de problemas de física se relaciona". Fue una sorpresa, en cierto modo, constatar las respuestas muy variadas que daban, ya que cada alumno asociaba el problema con distintos ejemplos de los tipos trabajados en clase.

Al mismo tiempo se ha podido comprobar la dificultad que supone para algunos estudiantes las variaciones y combinaciones de tipos de problemas, y también la gran diferencia entre el problema científico que propone el profesor y el que realmente entienden muchos alumnos, tal como ya mostraron Osborne y Freyberg (1991).

Paralelamente, los resultados de la aplicación de esta actividad de autoevaluación han comportado también cambios en el quehacer diario del profesor, obligándole a revisar sus rutinas para así ayudar mejor al alumnado a realizar este ejercicio metacognitivo. Un ejemplo de nueva práctica por parte del docente es que cada vez que se resuelve un problema en clase, después de leerlo atentamente y representarlo esquemáticamente, normalmente mediante un dibujo, se explicita la pregunta ¿A qué categoría o clase pertenece este problema? Se deja tiempo para la reflexión personal y después se discuten las respuestas, antes de continuar con la resolución. De esta manera se promueve que los alumnos realicen la conexión entre el problema que se está resolviendo y sus conocimientos. Así, son más capaces de autorregular su aprendizaje y mejorarlo, ya que pueden revisar, en cierta forma, la organización de su pensamiento y es sabido que el aprendizaje debe conducir a una organización interna de los conocimientos en estructuras cada vez más ordenadas y complejas (OÑORBE, 2003).

\section{Resultados y discusión}

El análisis de los resultados es muy alentador y positivo. Se ha constatado que las calificaciones de 33 de los 37 estudiantes estudiados son mejores después de aplicar la nueva metodología (Figura 1) y que la curva de Gauss de las calificaciones de los alumnos se ha desplazado hacia la derecha (Figura 2).

En la Figura 1 se presentan las calificaciones numéricas comparadas entre dos exámenes parciales que evaluaban la resolución de problemas de dinámica (en azul, antes de aplicar la nueva base de orientación mejorada con el análisis de la autoevaluación, y en rojo después). Y en la Figura 2, las curvas gaussianas de las calificaciones de los mismos exámenes. Se puede observar que la mejora es sustancial en todos los estudiantes que suspenden el primer parcial, lo cual nos puede indicar que estos alumnos han aprendido a autorregularse y que, posiblemente, 
los que obtenían buenos resultados ya eran capaces de hacerlo. Aun así, buena parte de estos últimos (60\% de los aprobados) también han mejorado sus resultados.

Figura 1. Calificaciones de los exámenes parciales de la segunda evaluación del curso 2011-2012

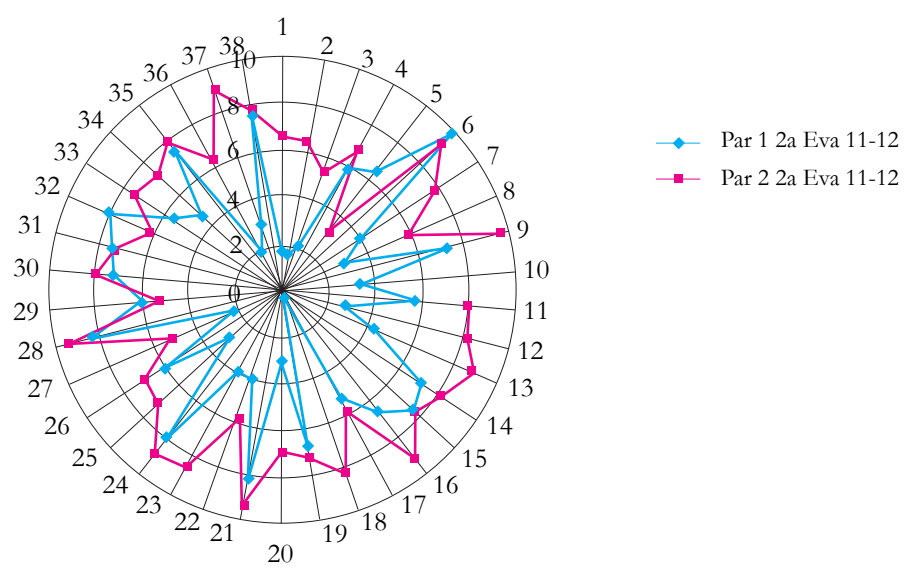

Fuente: elaborado por los autores

Figura 2. Distribución de las calificaciones de dos exámenes parciales del curso 20112012 según clases: 0 a 2,5 (2,5); 2,5 a 4,5 (4,5); 4,5 a 6,5 (6,5); 6,5 a 8,5 (8,5) i 8,5 a 10 (10)

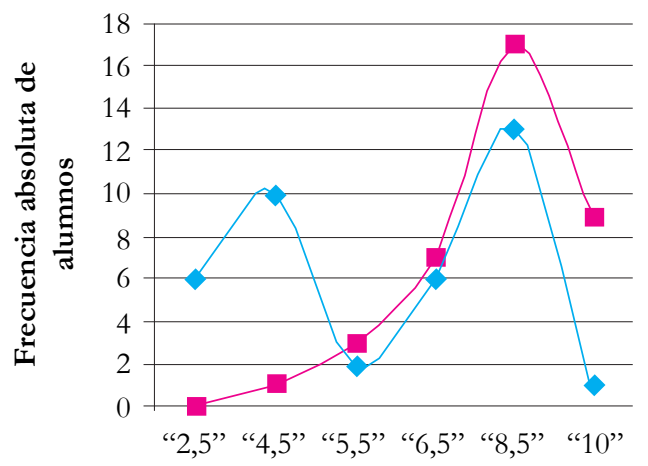

$\rightarrow \quad \operatorname{Par} 12 \mathrm{a}$ Eva 11-12

$\rightarrow \quad$ Par 2 2a Eva 11-12

Calificaciones

Fuente: elaborado por los autores. 
Para intentar objetivar estos resultados se ha establecido una comparación con los alumnos del curso pasado, ya que los resultados anteriores se podrían explicar sólo por el hecho de que en el segundo examen ya llevaban un tiempo trabajando este contenido. A continuación se presentan los resultados de los mismos parciales en el curso anterior (figuras 3 y 4). En estas figuras se puede observar que también hay una mejora aunque no tan significativa. Prueba de ello es que si hacemos la media de la mejora de un parcial al otro en el curso 2010-11 es de 1,2 mientras que la del curso 2011-12 asciende a 2,1.

También se ha buscado comprobar si estas diferencias se podrían explicar por el hecho de que el grupo de estudiantes del curso anterior fuera de un nivel más bajo que el del segundo año. Para ello se ha calculado la nota media de todos los alumnos de cada curso en la primera evaluación, siendo de 5,4 para los del curso 10-11 i de 4,7 para los del curso 11-12, por lo que es evidente que los buenos resultados del segundo año se explican por la manera en que se promovió la autorregulación. Este hallazgo apoya estudios previos que propusieron el papel positivo de la metacognición en el aprendizaje de la ciencia (DAVIS, 1996; THOMAS; McROBBIE, 2001).

Diversos alumnos han valorado esta mejora en las clases de resolución de problemas literalmente como "definitiva" y la aplican sistemáticamente cuando realizan sus tareas diarias, tanto en el aula como en casa.

Figura 3. Calificaciones de los exámenes parciales de la segunda evaluación del curso 2010-2011

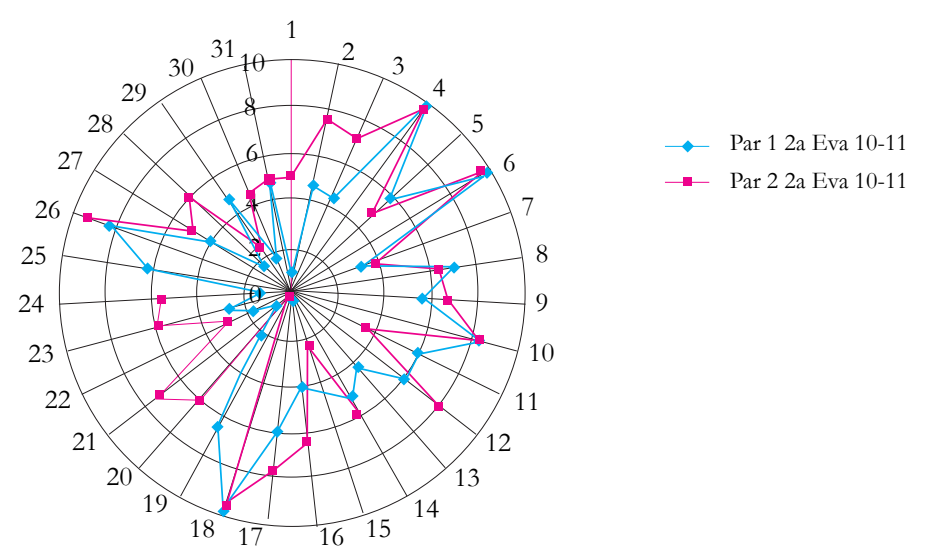

Fuente: elaborado por los autores 
Figura 4. Distribución de las calificaciones de dos exámenes parciales del curso 20102011 según las mismas clases que se muestran en la Figura 2

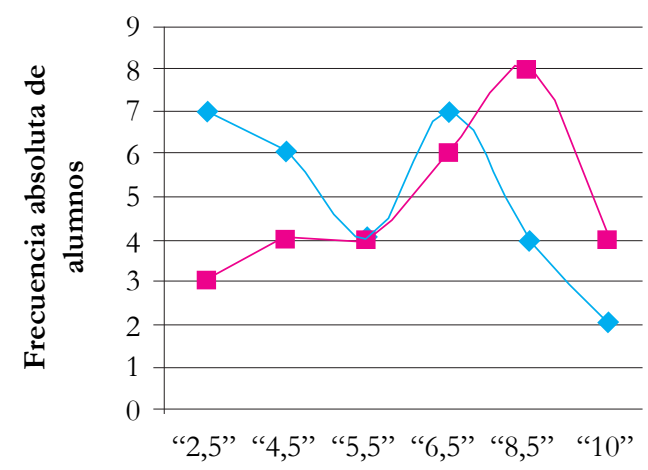

$\longrightarrow \quad \operatorname{Par} 12 \mathrm{a}$ Eva 10-11

$\rightarrow$ Par 2 2a Eva 10-11

Calificaciones

Fuente: elaborado por los autores.

Algunos alumnos critican la efectividad de la base de orientación para resolver problemas: "si sigues el protocolo [base de orientación] al pie de la letra, realizando todos los pasos, no acabarás a tiempo". Generalmente son alumnos que ya la tienen interiorizada de forma más resumida por lo que no la necesitan desarrollada. Pero otros aseguran que es de gran ayuda: "si no sigo el protocolo quizás me equivoco al principio y ya no habrá nada que hacer aunque acabe a tiempo"; "el protocolo te ayuda a no dejarte nada y pensar en todo"; "te ayuda a pensar y también a ver tus errores". Como dicen los estudiantes, "lo importante es saber de qué va el problema, o sea, responder la pregunta". Para conseguir este objetivo reconocen que necesitan enfrentarse a numerosos problemas y que los han de resolver poco a poco. Una alumna reflexiona así en su autoevaluación a la vista de sus errores: "¿Cómo arreglarlo? Haciendo más problemas en casa. Asi tendría más ejemplos para identificar los nuevos problemas y sería más fácil planificar las resoluciones". Estas reflexiones muestran que los estudiantes con dificultades son los que más valoran el interés de utilizar la base de orientación (y los criterios de autoevaluación asociados) ya que, como su nombre indica, les orienta y perciben que les ayuda a afrontarlas.

Ciertos alumnos nos han indicado, después de un año, que todavía utilizan dichos instrumentos para resolver problemas, incluso en otras asignaturas y con resultados satisfactorios para ellos.

\section{A modo de conclusiones}

En este estudio se ha podido comprobar que si se dan a los estudiantes pautas para reflexionar sobre el proceso que aplican para resolver problemas y sobre cómo activan los saberes aprendidos, apoyándose en estrategias metacognitivas, su percepción general es la de 
que entienden mejor los procesos de resolución y cómo aprenden, y que ello se traduce en mejores aprendizajes. También hemos podido observar que estas pautas les ayudan a ser más autónomos y, como es sabido, la autonomía es una de las principales características de los estudiantes que tienen éxito escolar o de los expertos en una materia determinada al realizar una tarea (SANMARTÍ; JORBA, 1995).

En otros trabajos (HINOJOSA; SANMARTÍ, 2015; ROSA; ALVES FILHO, 2014; SCHRAW; CRIPPEN; HARLEY, 2006) también se ha podido comprobar que trabajar los procesos metacognitivos con los alumnos, ayudándolos a tomar consciencia de su conocimiento y de sus estructuras conceptuales, mejora tanto su implicación con el aprendizaje como los resultados metodológicos y conceptuales. Y en concreto tal como muestran los resultados, son un buen camino para mejorar el proceso de resolución de problemas y la competencia de aprender a aprender.

El ambiente de enseñanza-aprendizaje creado en el aula a través de la implementación de dichos procesos metacognitivos marca un contraste directo con las prácticas pedagógicas que simplemente requieren escuchar y memorizar fórmulas y explicaciones sobre hechos del mundo físico, tal y como ya destacaban Hewson y Beeth (1995). A nivel afectivo el alumno interpreta estas estrategias metacognitivas como un acto de compromiso del profesorado por su formación. Pero este funcionamiento solo se puede lograr cuando las ideas, presuposiciones y sentimientos de los estudiantes se convierten en una parte integral del quehacer diario del aula, es decir, se pasa de una enseñanza centrada en el profesor a otra centrada en el alumno (POTVIN et al., 2010).

En cuanto a las a las propuestas para superar las dificultades que explicitan los estudiantes, reconocen que se ha de establecer una prioridad, es decir, que cada alumno que detecta problemas en relación a distintos ítems, tiene que decidir (si hace falta, aconsejado) qué tipo de obstáculo aborda primero. Y cuando lo ha solucionado, puede afrontar otro. Se ha comprobado que así el estudiante percibe que las dificultades son abordables y no se desanima tan fácilmente.

Otra consecuencia que merece un estudio más detallado, es que esta práctica parece espolear la motivación por el trabajo constante de los estudiantes con más dificultades. Por ejemplo, se dan cuenta de que los compañeros con mejores resultados siempre tienen respuesta a la pregunta sobre la categoría del problema, y que ello es una causa de éxito. Ello les anima a pensar en esta variable y a afrontar la resolución de problemas en casa, por lo que aumenta las horas de estudio (seguramente debido a que perciben que éste es un paso necesario y alcanzable en su proceso de aprendizaje). Estos tipos de procesos metaconceptuales, posiblemente, pueden mejorar la motivación de los estudiantes para aprender las entidades conceptuales desconocidas o no comprendidas (YURUK; BEETH; ANDERSEN, 2009).

\section{Agradecimientos}

Investigación realizada en el marco del grupo LiEC (Llenguatge i Ensenyament de les Ciències), grupo de investigación consolidado (referencia 2014SGR1492) por AGAUR (Agència de Gestió d'Ajuts Universitaris i de Recerca) y financiada por el Ministero de Educación y Ciencia (referencia EDU-2012-38022-C02-02). A la Escola Pia Sarrià-Calassanç. 


\section{Referencias}

BUNGE, M. La ciencia: su método y su filosofía. Pamplona: Editorial Laetoli, 2013.

BUTELER, L.; GANGOSO, Z. Algunos aspectos metodológicos de la investigación en resolución de problemas en física: una revisión. Ciência $\boldsymbol{\&}$ Educação, Bauru, v. 14, n. 1, p. 1-14, 2008. Disponible en: <http://dx.doi.org/10.1590/S1516-73132008000100001>. Acceso el: 23 nov. 2015.

CHI, M. T. H. Two approaches to the study of experts'characteristics. In: ERICSSON, K. A. et al. (Ed.). The Cambridge handbook of expertise and expert performance. New York: Cambridge University Press, 2006. p. 21-30.

CHI, M. T. H.; FELTOVICH, P. J.; GLASER, R. Categorization and representation of physics problems by experts and novices. Cognitive Science, Leiden, v. 5, n. 2, p. 121-152, 1981.

COOK, T. D.; REICHARDT, C. S. Qualitative and quantitative methods in evaluation research. London: Sage, 1986.

DAVIS, E. A. Metacognitive scaffolding to foster scientific explanations. In: ANNUAL MEETING OF THE AMERICAN EDUCATIONAL RESEARCH ASSOCIATION, 1996, New York. Disponible en: <http://files.eric.ed.gov/fulltext/ED394853.pdf>. Acceso el: 23 nov. 2015.

DUFRESNE, R. J. et al. ASK-IT/A2L: assessing student knowledge with instructional technology. Amherst: University of Massachusetts, Physics Education Research Group, 2000. (UMPERG technical report, PERG-2000\#09-SEP\#). Disponible en: <http://www. srri.umass.edu/sites/srri/files/dufresne-2000ask.pdf>. Acceso el: 23 nov. 2015.

GERACE, W. J.; BEATTY, I. D. Teaching vs. learning: changing perspectives on problem solving in physics instruction. In: COMMON CONFERENCE OF THE CYPRUS PHYSICS ASSOCIATION AND GREEK PHYSICS ASSOCIATION, 9., 2005, Nicosia, Cyprus. A Disponible en: <http://arxiv.org/ftp/physics/papers/0508/0508131.pdf>. Acceso el: 23 nov. 2015.

GIL, D.; MARTÍNEZ-TORREGROSA, J.; SENENT, F. El fracaso en la resolución de problemas de fisica: una investigación orientada por nuevos supuestos. Enseñanza de las Ciencias, Barcelona, v. 6, n. 2, p. 131-146, 1988. Disponible en: <http://rua.ua.es/dspace/ handle/10045/33495>. Acceso el: 23 nov. 2015.

GIL, D. et al. ¿Tiene sentido seguir distinguiendo entre aprendizaje de conceptos, resolución de problemas de lápiz y papel y realización de prácticas de laboratorio? Enseñanza de las Ciencias, Barcelona, v. 17, n. 2, p. 311-320, 1999.

GÓMEZ-FERRAGUD, C. Estudios sobre el establecimiento de analogías en resolución de problemas de ciencias: efectos del contexto, la estructura y la familiaridad con los enunciados. 2014. Memoria (Doctorado en Didácticas específicas) - Universitat de València, València, 2014. Disponible en: <http://roderic.uv.es/handle/10550/35986>. Acceso el: 23 nov. 2015. 
GÓMEZ-FERRAGUD, C.; SOLAZ, J. J.; SANJOSÉ, V. Efectos de la similitud superficial y estructural sobre la transferencia a partir de análogos en problemas de alta y baja familiaridad: primeros resultados. Enseñanza de las Ciencias, Barcelona, v. 31, n. 1, p. 135-151, 2013.

GUISASOLA, J. et al. La resolución de problemas basada en el desarrollode investigaciones guiadas en cursos introductorios de física universitaria. Enseñanza de las Ciencias, Barcelona, v. 29, n. 3, p. 439-452, 2011.

HINOJOSA, J.; SANMARTÍ, N. La autorregulación metacognitiva como medio para facilitar la transferencia en mecánica. Revista Eureka sobre Enseñanza y Divulgación de las Ciencias, Cádiz, v. 12, n. 2, p. 249-263, 2015. Disponible en: <http://dialnet.unirioja.es/ servlet/articulo?codigo $=5016521>$. Acceso el: 23 nov. 2015.

HINOJOSA, J.; SANMARTÍ, N. Resolver problemas colaborativamente de forma virtual. Alambique: didáctica de las ciencias experimentales, Barcelona, n. 67, p. 103-108, 2011.

HEWSON, P. W.; BEETH, M. E. Enseñanza para un cambio conceptual: ejemplos de fuerza y movimiento. Enseñanza de las Ciencias, Barcelona, v. 13, n. 1, p. 25-35, 1995.

HEYWORTH R. M. Quantitative problems solving in science: cognitive factors and directions for practice. Education Journal, Crediton, v. 22, n. 1, p.13-30, 1998.

JONASSEN, D. Toward a design theory of problem-solving. Educational Technology: research and development, New York, v. 48, n. 4, p. 63-85, 2000.

LORENZO, M. The development, implementation, and evaluation of a problem solving heuristic. International Journal of Science and Mathematics Education, Dordrecht, v. 3, n. 1, p. 33-58, 2005. Disponible en: <http://dx.doi.org/10.1007/s10763-004-8359-7>. Acceso el: 23 nov. 2015.

McDERMOT'T, L.C. Students' conceptions and problem solving in mechanics. In: TIBERGHIEN, A.; JOSSEM, E. L.; BAROJAS, J. (Ed.). Connecting research in physics education with teacher education. [S.1.]: International Commission on Physics Education, 1998. Disponible en: <http://www.iupap-icpe.org/publications/teach1/ ConnectingResInPhysEducWithTeacherEduc_Vol_1.pdf>. Acceso el: 23 nov. 2015.

NUNZIATI, G. Pour construire un dispositif d'évaluation formatrice. Cahiers

Pédagogiques, Paris, n. 280, p. 47-64, 1990. Disponible en: <http://web.ac-toulouse.fr/ automne_modules_files/pDocs/public/r7102_61_nunziati.pdf>. Acceso el: 23 nov. 2015.

OÑORBE, A. Resolución de problemas. In: JIMÉNEZ-ALEXANDRE, M. P. (Coord.).

Enseñar ciencias. Barcelona: Graó, 2003. p. 73-93.

OSBORNE, R.; FREYBERG, P. (Ed.). E1 aprendizaje de las ciencias: implicaciones de la ciencia de los alumnos. Madrid: Narcea, 1991.

PERALES, F. J. (Ed.). La resolución de problemas. Madrid: Síntesis, 2000.

POLYA, G. (Ed.). Cómo plantear y resolver problemas. México: Trillas, 1987. 
POTVIN, P. et al. Problem-centered learning vs. teaching-centered learning in science at the secondary level: an analysis of the dynamics of doubt. Journal of Applied Research on Learning, Ottawa, v. 3, n. 5, p. 1-24, 2010. Disponible en: <http://en.copian.ca/library/ research/jarl/problem/problem.pdf>. Acceso el: 23 nov. 2015.

PUIG, L.; CERDÁN, F. Problemas aritméticos escolares. Madrid: Síntesis, 1988. (Colección Matemáticas cultura y aprendizaje). Disponible en: <http://www.uv.es/puigl/ libros.html>. Acceso el: 23 nov. 2015.

REIF, F. Acquiring an effective understanding of scientific concepts. In: WEST, L. H. T.; PINES, L. (Ed.). Cognitive structure and conceptual change. Orlando: Academic Press, 1985. p. 133-151.

REIF, F. Millikan lecture 1994: understanding and teaching important scientific thought processes. American Journal of Physics, Melville, v. 63, p. 17-32, 1995.

ROSA, C. W.; ALVES FILHO, J. P. Estudo da viabilidade de uma proposta didática metacognitiva para as atividades experimentais em física. Ciência \& Educação, Bauru, v. 20, n. 1, p. 61-81, 2014. Disponible en: <http://dx.doi.org/10.1590/1516-731320140010005>. Acceso el: 23 nov. 2015.

SÁNCHEZ, P. M. Las representaciones mentales en la resolución de problemas de mecánica clásica. 2011. Tesis (Doctorado) - Departamento de Psicología Evolutiva y de la Educación, Facultad de Psicología, Universidad Nacional de Educación a Distancia, España, 2011. Disponible en: < http:/ /e-spacio.uned.es/fez/eserv/tesisuned:Psicologia-Pmsanchez/ Documento.pdf>. Acceso el: 23 nov. 2015.

SANJOSÉ, V. et al. Dificultades algebraicas en la resolución de problemas por transferencia. Revista Electrónica de Enseñanza de las Ciencias, Vigo, v. 6, n. 3, p. 538-561, 2007. Disponible en: < http://www.saum.uvigo.es/reec/volumenes/volumen6/art4_vol6_n3.pdf>. Acceso el: 23 nov. 2015.

SANMARTÍ, N. (Ed). Evaluar para aprender: 10 ideas clave. Barcelona: Graó, 2007.

SANMARTÍ, N.; JORBA, J. Autorregulación de los procesos de aprendizaje y construcción de conocimientos. Alambique: didáctica de las ciencias experimentales, Barcelona, n. 4, p. 5967, 1995.

SANMARTÍ, N.; SIMON, M.; MARQUEZ, C. La evaluación como proceso de autorregulación: 10 años después. Alambique: didáctica de las ciencias experimentales, Barcelona, n. 48, p. 32-41, 2006.

SCHOENFELD, A. What's all the fuss about metacognition? In: science and mathematics education. New Jersey: Erlbaum, 1987. p. 189-215.

SCHRAW, G.; CRIPPEN, K. J.; HARLEY, K. Promoting self-regulation in science education: metacognition as part of a broader perspective on learning. Research in Science Education, Dordrecht, v. 36, n. 1, p. 111-139, 2006. 
SOLAZ-PORTOLÉS, J. J.; SANJOSÉ-LÓPEZ, V. Conocimientos y procesos cognitivos en la resolución de problemas de ciencias: consecuencias para la enseñanza. Magis: revista internacional de investigación en educación, Bogotá, v. 1, n. 1, p. 147-162, 2008. Disponible en: <http://www.redalyc.org/articulo.oa?id=281021687010>. Acceso el: 23 nov. 2015.

THOMAS, P. G.; MCROBBIE, C. J. Using a metaphor for learning to improve students' metacognition in the chemistry classroom. Journal of Research in Science Teaching, Hoboken, v. 38, n. 2, p. 222-259, 2001.

TRUYOL, M. E.; GANGOSO, Z. La selección de diferentes tipos de problemas de Física como herramienta para orientar procesos cognitivos. Investigaçoes em Ensino de Ciencias, Porto Alegre, v. 3, n. 15, p. 463-484, 2010.

YURUK, N.; BEETH, M. E.; ANDERSEN, C. Analyzing the effect of metaconceptual teaching practices on students' understanding of force and motion concepts. Research in Science Education, Dordrecht, v. 39, n. 4, p. 449-475, 2009. 